\title{
Crystal structure of glutamine amidotransferase from Pyrococcus horikoshii OT3
}

\author{
By Shintaro Maruoka, Woo Cheol Lee, Masayuki Kamo, \\ Norio Kudo, Koji NAgata, and Masaru TANOKURA ${ }^{\dagger)}$ \\ Graduate School of Agricultural and Life Sciences, The University of Tokyo, \\ 1-1-1 Yayoi, Bunkyo-ku, Tokyo 113-8657 \\ (Communicated by Masanori OtsuKa, M. J. A.)
}

\begin{abstract}
Glutamine amidotransferases (GATases) hydrolyze glutamine and generate ammonia. The glutamine amide nitrogen is utilized for the biosynthesis of a variety of molecules such as amino acids, coenzymes, antibiotics, purine and pyrimidine nucleotides, and glucosamine. Here, we determined the crystal structure of a GATase (PH1346) from the hyperthermophilic archaeon Pyrococcus horikoshii OT3 at $1.89 \AA$ A resolution. Its overall structure and active site are the most similar to those of $E$. coli guanosine 5'monophosphate (GMP) synthase and Sulfolobus solfataricus anthranilate synthase, respectively.
\end{abstract}

Key words: Glutamine amidotransferase; protein structure; X-ray crystallography.

Introduction. Glutamine amidotransferase (GATase) is an enzyme widespread in living organisms. It catalyzes the hydrolysis of glutamine to generate glutamic acid and ammonia. It works cooperatively with various synthases by providing ammonium nitrogen that is utilized to produce amino acids, purine and pyrimidine nucleotides, amino sugars, coenzymes and antibiotics. ${ }^{1)}$ Class-I GATase contains a characteristic Cys-His-Glu triad. The carbonyl carbon of the glutamine side chain carboxamide group is attacked by the thiolate anion of Cys, and forms a tetrahedral intermediate. Then the protonation of the amino group by His collapses this intermediate, producing a thioester intermediate and ammonia. ${ }^{1)}$ Three-dimensional structures of Class-I GATase have been reported in several species including an eukaryote, ${ }^{2)}$ ten bacteria ${ }^{3)-12)}$ and an archaeon. ${ }^{13)}$ Here, we report the crystal structure of a Class-I GATase from the hyperthermophilic archaeon Pyrococcus horikoshii OT3 (PH1346, a putative guanosine 5'-monophosphate (GMP) synthase, E.C. 6.3.5.2) ${ }^{14)}$ and its comparison with the crystal structures of anthranilate synthase from Sulfolobus solfataricus (34\% identical in sequence) and GMP synthase from $E$. coli (37\% identical in sequence).

Materials and methods. The GATase from $P$.

\footnotetext{
†) Correspondence to: M. Tanokura.
}

horikoshii (PH-GATase) was overexpressed in E. coli BL21(DE3) cells as a fusion protein with an N-terminal His tag using the pET-28a(+) vector (Novagen). The harvested cells were sonicated in $50 \mathrm{mM}$ sodium phosphate buffer (pH 8.0) containing $300 \mathrm{mM} \mathrm{NaCl}$ and $10 \mathrm{mM}$ imidazole and then centrifuged at $40,000 \mathrm{~g}$ for $30 \mathrm{~min}$. The supernatant was incubated for $30 \mathrm{~min}$ at $353 \mathrm{~K}$ and centrifuged at $40,000 \mathrm{~g}$ for $30 \mathrm{~min}$. The supernatant was batch-incubated with Ni-NTA-agarose (Qiagen) for 30 min and applied to an Econopac column (Bio-Rad, Hercules, CA). The column was washed with $50 \mathrm{mM}$ sodium phosphate buffer (pH 8.0) containing $300 \mathrm{mM}$ $\mathrm{NaCl}$ and $20 \mathrm{mM}$ imidazole. Then the protein was eluted in $50 \mathrm{mM}$ sodium phosphate buffer ( $\mathrm{pH}$ 8.0) containing $300 \mathrm{mM} \mathrm{NaCl}$ and $250 \mathrm{mM}$ imidazole. The protein was further purified by size exclusion chromatography with a HiLoad 26/60 Superdex 75 pg column (Amersham) equilibrated with $20 \mathrm{mM}$ Tris-HCl buffer (pH 8.0) containing $100 \mathrm{mM} \mathrm{NaCl}$. The fractions containing $\mathrm{PH}-$ GATase were dialyzed against 5 mM Tris-HCl ( $\mathrm{pH} 8.0$ ) and concentrated to $28.6 \mathrm{mg} / \mathrm{ml}$ in Centriprep-3 concentrators (Amicon). The purity was ascertained by SDSPAGE and the concentration was determined with the absorbance at $280 \mathrm{~nm}$.

Crystallization was carried out at $278 \mathrm{~K}$ by the hanging-drop vapor-diffusion method using Crystal Screens 1 and 2 (Hampton Research). Each hanging-drop was 


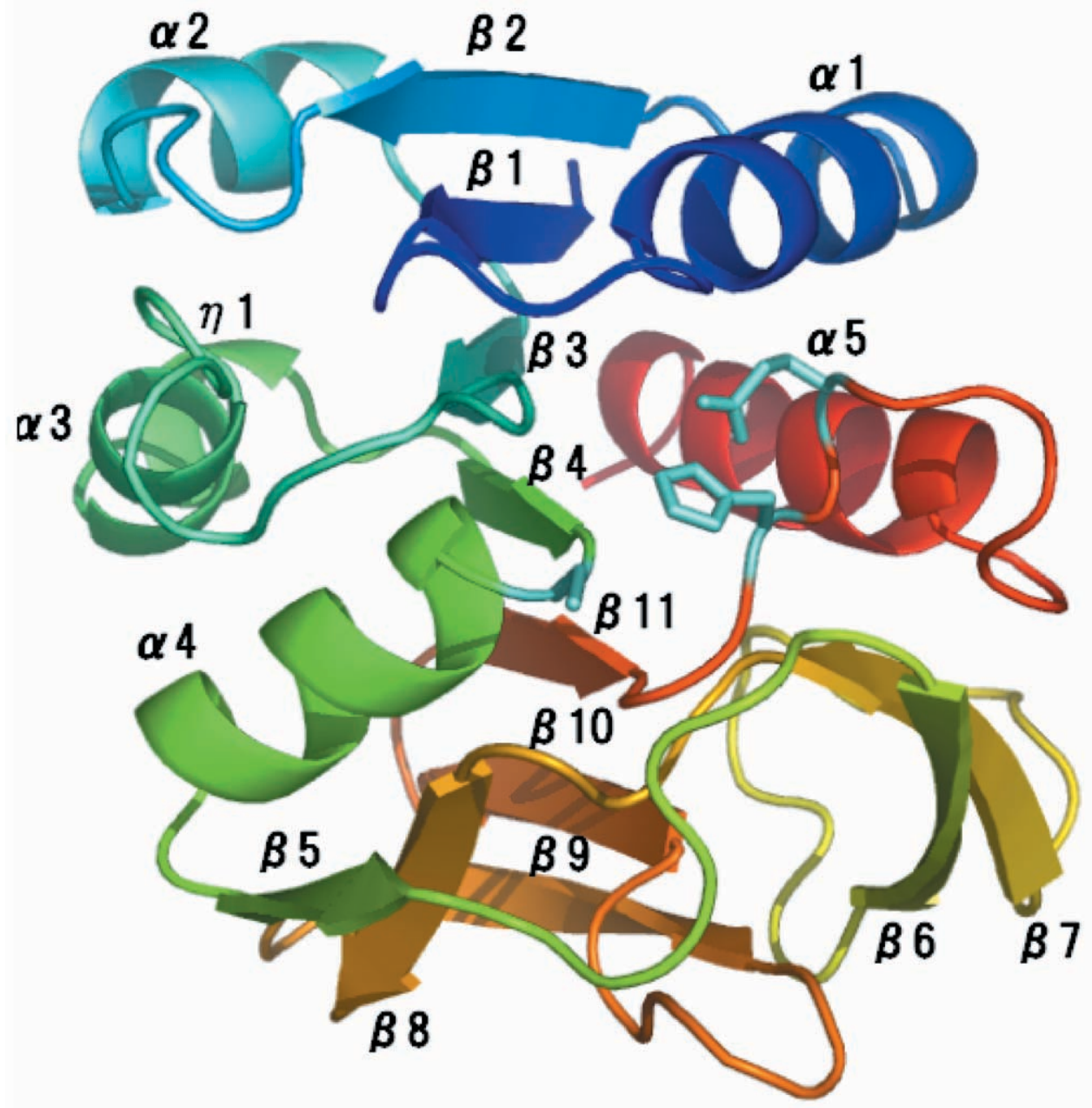

Fig. 1. The overall structure of PH-GATase. Color coding runs from blue at the N-terminal region to red at the C-terminal region. Secondary structure assignments are labeled on the ribbon model: $\alpha, \alpha$-helix; $\beta, \beta$-strand; and $\eta, 3_{10}$-helix. The location of the active site triad (Cys79-His166-Glu168) is represented in sticks shown in light blue near $\alpha 5$-helix.
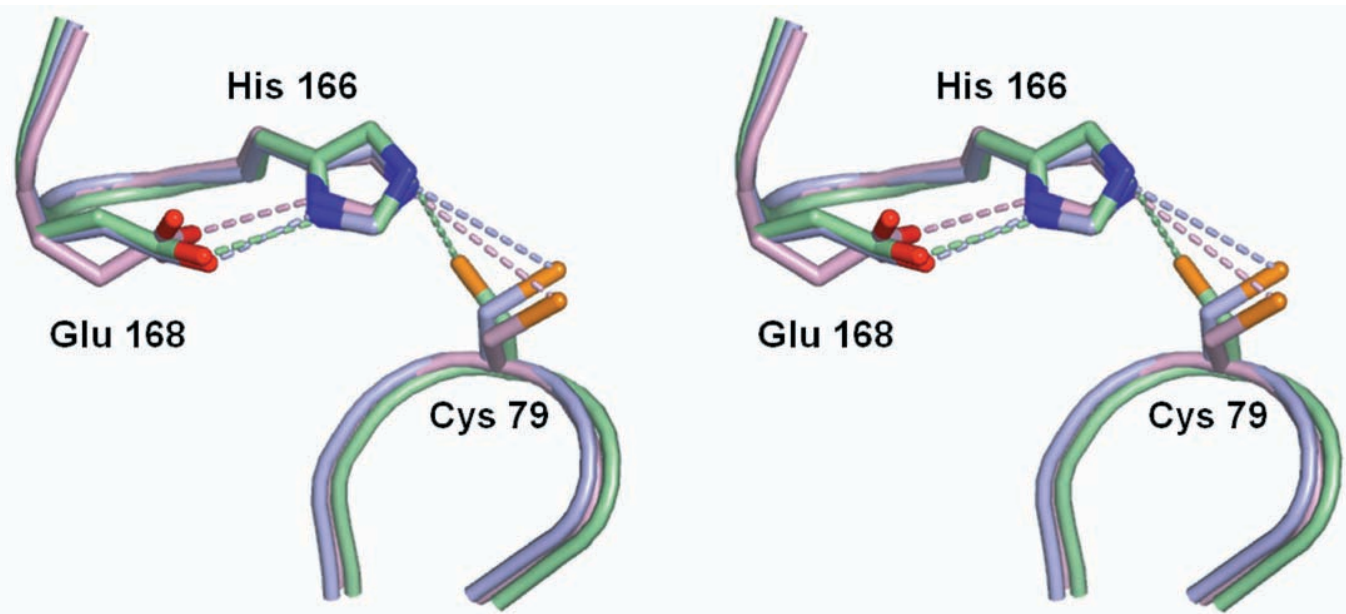

Fig. 2. Stereo diagram of the GATase active sites. PH-GATase (blue), GuaA (green), and TrpG (pink) are superposed. The active-site triad, Cys79, His166 and Glu168, are conserved in these molecules. The residue number corresponds to PH-GATase. 
prepared by mixing $2 \mu \mathrm{l}$ protein solution $(20 \mathrm{mg} / \mathrm{ml}$ in 5 $\mathrm{mM}$ Tris-HCl pH 8.0) with $2 \mu$ reservoir solution and equilibrated against $500 \mu \mathrm{l}$ of the reservoir solution. Crystals suitable for X-ray analysis were obtained in a few days with the reservoir solution consisting of $100 \mathrm{mM}$ sodium acetate $\mathrm{pH} 4.6$ and $2.0 \mathrm{M}$ sodium chloride. SeMet-labeled ${ }^{15)}$ PH-GATase was prepared with the same expression system and crystallized under the same conditions.

The crystals were soaked in the reservoir solution supplemented with $10 \%$ (v/v) glycerol as the cryoprotectant for a few seconds before flash-freezing in the cryo nitrogen gas stream. X-ray diffraction data were collected at beamline BL41XU at SPring-8 (Harima, Japan). The native dataset was collected at $0.9740 \AA$. The crystals diffracted X-rays beyond $1.60 \AA$ resolution. The diffraction data to $1.89 \AA$ were indexed and scaled with the programs HKL2000 and SCALEPACK, ${ }^{16)}$ respectively. The crystals belonged to space group $P 3_{1} 21$ or $P 3_{2} 21$ with unit cell dimensions $a=b=65.9 \AA$ and $c=116.4 \AA$. The crystal contained one protein molecule per asymmetric unit according to the Matthews coefficient $\left(V_{\mathrm{m}}=\right.$ $3.0 \AA^{3} \mathrm{Da}^{-1}$ ) and solvent content of $58.7 \%{ }^{17)}$ The datasets for a SeMet-labeled crystal were collected at wavelengths of $0.9894 \AA$ (remote), $0.9792 \AA$ (peak), and $0.9794 \AA$ (edge).

The structure of SeMet-labeled GATase was solved using the single-wavelength anomalous diffraction (SAD) method using the peak dataset. Five positions out of six Se atoms in an asymmetric unit were determined using the program SOLVE. ${ }^{18)}$ The program RESOLVE ${ }^{19)}$ was used to improve phases. The density map was obtained only when we used the space group $P 3_{2} 21$. Automated model building was carried out with $\mathrm{ARP} / \mathrm{wARP}^{20)}$ and the remainder was built manually with XtalView. ${ }^{21)}$ The structure was refined with Refmac5. ${ }^{22)}$ The structure of the native enzyme was then determined by the molecular replacement method with the program MOLREP ${ }^{23)}$ using the refined structure of SeMet-labeled PH-GATase as an initial model. Then, the structure was refined with the following programs: $\mathrm{CNS}^{24)}$ (simulated annealing and $B$-factor refinements), XtalView (manual fitting), and Refmac5 (TLS ${ }^{25)}$ and restrained refinements). The refined structure was validated with PROCHECK ${ }^{26)}$ and visualized with PyMOL. ${ }^{27}$ The atomic coordinates of PH-GATase have been deposited into the Protein Data Bank with the accession number of 2D7J.

Results and discussion. The crystal structure of PH-GATase was solved at $1.89 \AA$ A resolution and refined to an $R_{\text {factor }}$ of $18.6 \%$ and an $R_{\text {free }}$ of $21.0 \%$. The structure turned out to be a monomer. The final electron density allowed modeling of 187 residues as well as 53 water molecules. N-terminal His-tag, Met1, and the side chains of Asp112 and C-terminal Leu189 were missing. Asp112 and Leu189 were modeled as Ala. The backbone geometry of the native structure was ascertained by Ramachandran plot, where $89.9 \%$ and $8.2 \%$ of the residues were in the most favored and additional allowed regions, respectively. Cys79 and Asp112 (modeled as Ala) were in the disallowed regions. The activesite Cys79 had an unusual backbone torsion angles ( $\varphi=$ $55.9^{\circ}, \Psi=102.5^{\circ}$ ), but that is a characteristic feature of this class of enzymes. ${ }^{12)}$

The overall structure of PH-GATase is shown in Fig. 1. PH-GATase has an $\alpha / \beta$ structure consisting of $11 \beta$ strands, five $\alpha$-helices and one $3_{10}$-helix. The core of the domain is a seven-stranded $\beta$-sheet $(\beta 1, \beta 2, \beta 3, \beta 4, \beta 9$, $\beta 10, \beta 11$ ) surrounded by $\alpha$-helices on both sides.

Two closest structural homologues to PH-GATase were found in the DALI database. ${ }^{28)}$ One was the glutaminase subdomain (GuaA) of GMP synthase from $E$. coli $(\mathrm{PDB} \text { code } 1 \mathrm{GPM})^{3)}$ and the other was the glutaminase subunit (TrpG) of anthranilate synthase from Sulfolobus solfataricus (PDB code 1QDL). ${ }^{13)}$ The sequence identities and root-mean-square deviations (RMSDs) were $37 \%$ and $1.8 \AA$ (for $182 \mathrm{C}^{\alpha}$ atoms) between PH-GATase and GuaA, and 34\% and $1.6 \AA$ (for $179 \mathrm{C}^{\alpha}$ atoms) between PH-GATase and TrpG.

The superposition of the active sites of these molecules are shown in Fig. 2. The residues of the active-site triad consisting of Cys79, His166 and Glu168 are well conserved among the three enzymes. But there are two remarkable differences in the side-chain conformation of the active-site residues. The side-chain conformation of Glu168 is different between PH-GATase/GuaA and TrpG, but this difference does not affect the hydrogen bond between Glu168 and His 166. On the other hand, the Cys $\mathrm{C}^{\beta}-\mathrm{S}^{\gamma}$ bond in Cys79 is rotated ca. $100^{\circ}$ between PH-GATase/TrpG and GuaA, which suggests that the substrate recognition of $\mathrm{PH}-\mathrm{GAT}$ Tase would be closer to that of TrpG than that of GuaA. We are now trying to crystallize PH-GATase in complex with substrate (glutamine) or inhibitor to analyze the structural basis of substrate recognition and reaction mechanism of PH-GATase.

Acknowledgments. We would like to express gratitude to Drs. Kawamoto and Sakai at RIKEN Harima Institute for their kind help in data collection. The synchrotron-radiation experiments were performed at BL41XU at SPring-8 with the approval of the 
Japan Synchrotron Radiation Research Institute (Proposal 2003A0565-NL1-np). This work was supported in part by the National Project on Protein Structural and Functional Analyses of the Ministry of Education, Culture, Sports, Science and Technology of Japan and by Grants-in-Aid for Scientific Research from the Ministry of Education, Culture, Sports, Science and Technology of Japan.

\section{References}

1) Zalkin, H. (1993) Adv. Enzymol. Relat. Areas Mol. Biol. 66, 203-309.

2) Chaudhuri, B. N., Lange, S. C., Myers, R. S., Chittur, S. V., Davisson, V. J., and Smith, J. L. (2001) Structure 9, 987-997.

3) Tesmer, J. J. G., Klem, T. J., Deras, M. L., Davisson, V. J., and Smith, J. L. (1996) Nat. Struct. Biol. 3, 74-86.

4) Thoden, J. B., Holden, H. M., Wesenberg, G., Raushel, F. M., and Rayment, I. (1997) Biochemistry 36, 6305-6316.

5) Endrizzi, J. A., Kim, H., Anderson, P. M., and Baldwin E. P. (2004) Biochemistry 43, 6447-6463.

6) Morollo, A. A., and Eck, M. J. (2001) Nat. Struct. Biol. 8, 243-247.

7) Spraggon, G., Kim, C., Nguyen-Huu, X., Yee, M. C., Yanofsky, C., and Mills, S. E. (2001) Proc. Natl. Acad. Sci. USA 98, 6021-6026.

8) Omi, R., Mizuguchi, H., Goto, M., Miyahara, I., Hayashi, H., Kagamiyama, H., and Hirotsu, K. (2002) J. Biochem. 132, 759-765.

9) Douangamath, A., Walker, M., Beismann-Driemeyer, S., Vega-Ferenandez, M. C., Sterner, R., and Wilmanns, M. (2002) Structure 10, 185-193.

10) Chen, S., Tomchick, D. R., Wolle, D., Hu, P., Smith, J. L., Switzer, R. L., and Zalkin, H. (1997) Biochemistry 36, 10718-10726.

11) Muchmore, C. R., Krahn, J. M., Kim, J. H., Zalkin, H., and Smith, J. L. (1998) Protein Sci. 7, 39-51.
12) Korolev, S., Skarina, T., Evdokimova, E., Beasley, S., Edwards, A., Joachimiak, A., and Savchenko, A. (2002) Proteins 49, 420-422.

13) Knochel, T., Ivens, A., Hester, G., Gonzalez, A., Bauerle, R., Wilmanns, M., Kirschner, K., and Jansonius, J. N. (1999) Proc. Natl. Acad. Sci. USA 96, 9479-9484.

14) Kawarabayasi, Y., Sawada, M., Horikawa, H., Haikawa, Y., Hino, Y., Yamamoto, S., Sekine, M., Baba, S.-I., Kosugi, H., Hosoyama, A. et al. (1998) DNA Res. 5, 55-76.

15) Van Duyne, G. D., Standaert, R. F., Karplus, P. A., Schreiber, S. L., and Clardy, J. (1993) J. Mol. Biol. 229, 105-124.

16) Otwinowski, Z., and Minor, W. (1997) Methods Enzymol. 276, $307-326$.

17) Matthews, B. W. (1968) J. Mol. Biol. 33, 491-497.

18) Terwilliger, T. C., and Berendzen, J. (1999) Acta Cryst. D55, 849-861.

19) Terwilliger, T. C. (2000) Acta Cryst. D56, 965-972.

20) Perrakis, A., Morris, R., and Lamzin, V. S. (1999) Nat. Struct. Biol. 6, 458-463.

21) McRee, D. E. (1999) J. Struct. Biol. 125, 156-165.

22) Murshudov, G. N., Vagin, A. A., and Dodson, E. J. (1997) Acta Cryst. D53, 240-255.

23) Vagin, A. A., and Teplyakov, A. (1997) J. Appl. Cryst. 30, 1022-1025.

24) Brünger, A. T., Adams, P. D., Clore, G. M., DeLano, W. L., Gros, P., Grosse-Kunstleve, R. W., Jiang, J.-S., Kuszewski, J., Nilges, M., Pannu, N. S. et al. (1998) Acta Cryst. D54, 905-921.

25) Painter, J., and Merritt, E. (2005) Acta Cryst. D61, 465-471.

26) Laskowski, R. A., MacArthur, M. W., Moss, D. S., and Thornton, J. M. (1993) J. Appl. Cryst. 26, 283-291.

27) DeLano, W. L. (2002) http://www.pymol.org/.

28) Holm, L., and Sander, C. (1993) J. Mol. Biol. 233, 123-138.

(Received Nov. 23, 2005; accepted Dec. 12, 2005) 